\title{
FLAVONAS, LIGNANAS E TERPENO DE Piper umbellata (PIPERACEAE)
}

\author{
Debora Cristina Baldoqui, Vanderlan da S. Bolzani e Maysa Furlan* \\ Instituto de Química, Universidade Estadual Paulista, CP 355, 14800-900 Araraquara - SP, Brasil \\ Massuo J. Kato \\ Instituto de Química, Universidade de São Paulo, CP 26077, 05599-970 São Paulo - SP, Brasil \\ Márcia O. M. Marques \\ Instituto Agronômico de Campinas, 13075-630 Campinas - SP, Brasil
}

Recebido em 14/1/08; aceito em 10/12/08; publicado na web em 7/5/09

\begin{abstract}
FLAVONES, LIGNANS AND TERPENE FROM Piper umbellata (PIPERACEAE). The phytochemical investigation of Piper umbellata leaves yielded nine compounds including one terpenoid glucoside, five flavones (vitexin 2"-O- $\beta$-glucopyranoside, apigenin 8-C- $\beta$-D-glucopyranoside, orientin 8-C- $\beta$-D-glucopyranoside, 5-hydroxy-7,3',4'-trimethoxy-flavone and velutin), two lignans (sesamin e dihydrocubebin) and 4-nerolidylcathecol. Excepting 4-nerolidylcathecol, all compounds have not been described from this species yet.
\end{abstract}

Keywords: Piper umbellata; Potomorphe umbellata; constitutive metabolites.

\section{INTRODUÇÃo}

Piperaceae, que pertence à ordem Piperales, tem sido classificada junto com Chlorantaceae entre as mais primitivas angiospermas podendo, assim, ser considerada um fóssil vegetal vivo. ${ }^{1}$ A grande maioria de suas espécies produz frutos do tipo espigas com grande quantidade de sementes que possuem síndrome de dispersão zoocórica, sendo muito freqüente a dispersão por morcegos. ${ }^{2}$

Espécies de Piperaceae têm sido utilizadas na alimentação (como condimento - Piper nigrum),${ }^{3}$ como inseticidas ${ }^{4}$ e, também, na medicina tradicional devido ao acúmulo de diferentes classes de metabólitos biologicamente ativos, tais como fenilpropanóides, ${ }^{5}$ lignanas/neolignanas, ${ }^{6-10}$ pironas, ${ }^{11}$ amidas alífáticas e aromáticas, ${ }^{12-14}$ alcalóides, ${ }^{15}$ policetídeos ${ }^{16}$ e cromenos, ${ }^{17-22}$ além de outros metabólitos de biossíntese mista. ${ }^{7,23,24}$

As espécies de Piperaceae estão sendo subdivididas em quatro gêneros ${ }^{25}$ sendo que os mais abundantes são Peperomia Ruiz e Pavon, com aproximadamente 1700 espécies $^{26}$ e Piper L., com cerca de 2000 espécies. ${ }^{27}$ Muitos gêneros foram incluídos como sinonímias dentro do gênero Piper, por exemplo, Arctottonia Trel., Macropiper Miq., Pothomorphe Miq., Ottonia Spreng., e Trianaeopiper Trel. ${ }^{28}$

A espécie Piper umbellata, anteriormente classificada como Potomorphe umbellata ${ }^{29}$ conhecida popularmente no Brasil como pariparoba, caapeba, capeba e malvisco é uma planta arbustiva, de ocorrência pantropical, que chega a medir aproximadamente $3 \mathrm{~m}$ de altura. ${ }^{29}$ Esta planta é utilizada na medicina popular no tratamento de doenças do fígado, epilepsia e como antimalárico.$^{30}$ Estudos biológicos demonstraram que possui atividade antimalárica e antiinflamatória. ${ }^{30-32}$

Além disso, recentes estudos demonstraram que apresenta atividade protetora contra raios ultravioleta do tipo UVB,,$^{33}$ o que provavelmente está relacionada com o 4-nerolidilcatecol, componente majoritário nas folhas e raízes desta espécie, uma vez que este possui atividade antioxidante mais potente que a do alfa-tocoferol (vitamina E), a qual é utilizada em formulações cosméticas para a prevenção do envelhecimento cutâneo. Estes relatos mostram a importância deste metabólito secundário do ponto de vista comercial, uma vez que formulações contendo extrato de Piper umbellata têm sido empregadas em produtos de uso cosmético, tais como gel, creme e filtro solar.

\footnotetext{
*e-mail: maysaf@iq.unesp.br
}

Sendo assim, o conhecimento dos demais metabólitos presentes nesta espécie é importante, e o presente trabalho descreve o isolamento dos principais metabólitos secundários que ocorrem nas folhas de Piper umbellata, visto que os trabalhos descritos na literatura sobre esta espécie reportam somente o isolamento do 4-nerolidilcatecol, ${ }^{29}$ incluindo o estudo de sua potencialidade biológica ${ }^{30,32,34,35}$ e estudos biossintéticos. ${ }^{36,37}$

\section{PARTE EXPERIMENTAL}

\section{Material vegetal}

As folhas e raízes de Piper umbellata foram coletadas em novembro de 1999, na Universidade de São Paulo, e sua identificação foi feita pelo Dr. G. E. D. Paredes (Universid Pedro Ruiz Gallo - Peru), sendo que exsicatas (Kato-671) estão depositadas no Instituto de Biociências da USP, São Paulo.

As folhas utilizadas na extração de substâncias ácidas e na obtenção do óleo essencial foram coletadas de um espécime adulto cultivado nas dependências do Instituto de Química da UNESP de Araraquara.

\section{Extração dos componentes não voláteis}

As folhas de Piper umbellata (2000 g) secas e trituradas foram submetidas à extração por maceração com etanol. O extrato etanólico (225,0 g) foi dissolvido em MeOH:H $\mathrm{H}_{2} \mathrm{O}$ na proporção 4:1 e submetido a extrações líquido-líquido com hexano, $\mathrm{CH}_{2} \mathrm{Cl}_{2}$ e AcOEt.

A fração $\mathrm{AcOEt}(8,0 \mathrm{~g})$ foi submetida a uma partição em BuOH: $\mathrm{H}_{2} \mathrm{O}$ (1:1). O extrato butanólico obtido foi concentrado em rotaevaporador (2,0 g) e aplicado em uma coluna de Sephadex LH 20 eluída com MeOH (100\%) em um fluxo de $0,53 \mathrm{~mL} / \mathrm{min}$, resultando em 206 frações de 8 $\mathrm{mL}$. As frações obtidas foram analisadas por CCDC utilizando como fase móvel BuOH:MeOH: $\mathrm{H}_{2} \mathrm{O}$ (60:15:25) e agrupadas em 17 frações. A fração $5(0,134 \mathrm{~g})$ foi identificada como a substância 1 (rel-(6S, 9S)roseosídeo). A fração 8 foi recristalizada em $\mathrm{MeOH}$ e resultou em um pó branco solúvel em DMSO, identificada como sendo a substância 2 (C-glicosilflavona-O-glicosídeo, vitexina 2"-O- $\beta$-D-glucopiranosídeo) $(0,212 \mathrm{~g})$. A fração 12 também foi recristalizada em $\mathrm{MeOH}$ e resultou em um pó amarelo identificado como sendo a substância 3 (C-glicosilflavonas, apigenina-8-C- $\beta$-D-glucopiranosídeo) $(0,007$ g). O mesmo 
<smiles>CC1=CC(=O)CC(C)(C)[C@@]1(O)/C=C/C(C)O</smiles>

1

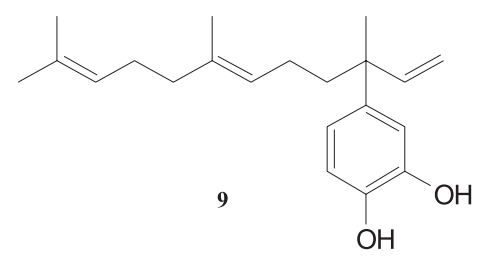<smiles>[R]c1ccc(C2CC(=O)c3c(O)cc([R2])c([R])c3O2)cc1[R3]</smiles>

\begin{tabular}{c|cccc} 
& $\mathbf{R}_{\mathbf{1}}$ & $\mathbf{R}_{\mathbf{2}}$ & $\mathbf{R}_{\mathbf{3}}$ & $\mathbf{R}_{\mathbf{4}}$ \\
\hline $\mathbf{2}$ & C-Gli-O-Gli & $\mathrm{OH}$ & $\mathrm{H}$ & $\mathrm{OH}$ \\
$\mathbf{3}$ & $\mathrm{C}-\mathrm{Gli}$ & $\mathrm{OH}$ & $\mathrm{H}$ & $\mathrm{OH}$ \\
$\mathbf{4}$ & $\mathrm{C}-\mathrm{Gli}$ & $\mathrm{OH}$ & $\mathrm{OH}$ & $\mathrm{OH}$ \\
$\mathbf{6}$ & $\mathrm{H}$ & $\mathrm{OCH}_{3}$ & $\mathrm{OCH}_{3}$ & $\mathrm{OH}$ \\
$\mathbf{7}$ & $\mathrm{H}$ & $\mathrm{OCH}_{3}$ & $\mathrm{OCH}_{3}$ & $\mathrm{OCH}_{3}$
\end{tabular}

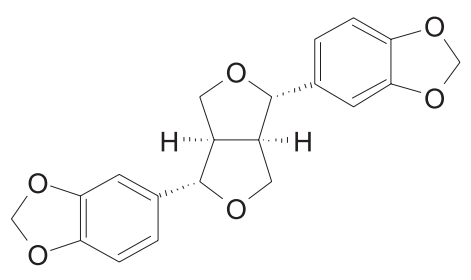

5

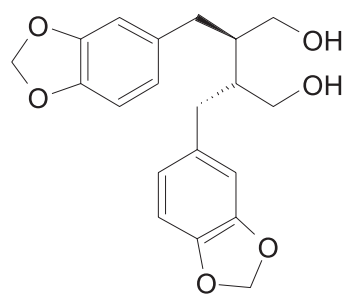

8
Figura 1. Estruturas químicas das substâncias isoladas das folhas de Piper umbellata

procedimento foi utilizado para a purificação da fração 14, que resultou em um pó amarelo, a qual foi identificada como sendo substância 4 (orientina 8-C- $\beta$-D-glucopiranosídeo) (0,006 g).

A fração $\mathrm{CH}_{2} \mathrm{Cl}_{2}(18,0 \mathrm{~g})$ foi submetida à $\mathrm{CC}$ de sílica gel 60 , eluída com Hex:AcOEt, em proporções crescentes de polaridade, resultando em 13 frações. A fração 4 (0,235 g) foi recristalizada em hexano e resultou em um precipitado em forma de agulhas, o qual foi identificado como sendo a substância 5 (sesamina) $(0,183$ g). A fração 8 (0,488 g) foi submetida a uma coluna de sílica gel eluída com $\mathrm{CHCl}_{3}: \mathrm{MeOH}$ em proporções crescentes de polaridade, resultando em 21 frações. A fração 8-2 (0,020 g) foi submetida à CCDP, eluída com $\mathrm{CHCl}_{3}: \mathrm{MeOH}(95: 5)$, fornecendo 2 frações. A fração 8-2-1 resultou em um pó amarelo, o qual foi identificado como sendo a substância 6 (5-hidroxi-7,3',4'-trimetoxiflavona ) $(0,006 \mathrm{~g})$. A fração 8-5 (0,052 g) foi submetida à CCDP, eluída com $\mathrm{CHCl}_{3}: \mathrm{MeOH}$ (95:5), fornecendo 3 frações. A fração 8-5-1, um pó amarelo, foi identificada como sendo a substância 7 (velutina) $(0,008$ g). A fração $10(0,931 \mathrm{~g})$ foi submetida a uma coluna de sílica eluída com $\mathrm{CHCl}_{3}: \mathrm{MeOH}$ em proporções crescentes de polaridade, resultando em 20 frações. A fração 10-13 (0,102 g) foi submetida a uma coluna de

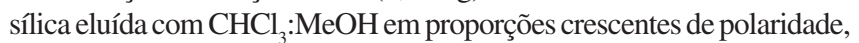
fornecendo 15 frações. A fração 10-13-6 foi identificada como sendo a substância 8 (diidrocubebina) $(0,024 \mathrm{~g})$.

O último extrato estudado foi o extrato hexânico (166 g) e levou ao reisolamento das substâncias $\mathbf{5}$ e $\mathbf{6}$, além do isolamento 4-nerolidilcatecol (9) já isolado previamente de folhas de Piper umbellata.

\section{RESULTADOS E DISCUSSÃO}

O estudo fitoquímico das folhas de Piper umbellata levou ao isolamento de 9 substâncias, as quais foram identificadas pela análise de dados espectrométricos, tais como RMN de ${ }^{1} \mathrm{He}{ }^{13} \mathrm{C}$, HMQC, HMBC e EM. Excetuando-se o 4-nerolidilcatecol, todas as substâncias isoladas neste trabalho são descritas pela primeira vez nesta espécie vegetal.

A substância 1 foi identificada como sendo um roseosídeo, um terpeno glicosilado primeiramente isolado de folhas de Vinca rosea ${ }^{38}$ Comparando os dados de $\mathrm{RMN}$ de ${ }^{13} \mathrm{C}$ com os publicados na literatu$\mathrm{ra}^{39,40}$ foi possível propor a estereoquímica relativa da substância $\mathbf{1}$ como sendo rel-(6S, 9S)-roseosídeo, devido aos deslocamentos químicos dos carbonos C-6, C-7, C-8 e C-9 em $\delta$ 78,0, 131,7, 131,7 e 72,0, respectivamente, os quais são significantemente diferentes dos deslocamentos químicos na estrutura do $(6 S, 9 R)$-roseosídeo ${ }^{39,40} \mathrm{em} \delta 80,0,134,8,131,4$ e 76,2, respectivamente. O estereoisômero $(6 S, 9 R)$ já foi isolado de Piper elongatum VAHL, ${ }^{41}$ entretanto, o estereoisômero $(6 S, 9 S)$ está sendo descrito pela primeira vez em espécies de Piper.

As substâncias 2-4,6 e 7 foram identificadas como sendo flavonas, através da análise dos dados de EM, RMN de ${ }^{1} \mathrm{H}$ e de ${ }^{13} \mathrm{C}$ e espectros bidimensionais COSY ${ }^{1} \mathrm{H}-{ }^{1} \mathrm{H}, \mathrm{HMQC}$ e HMBC, além de comparação com dados publicados anteriormente. Nos espectros de RMN de ${ }^{1} \mathrm{H}$ as flavonas mostram um singleto entre $\delta$ 6,0 e $\delta$ 8,0 característico do hidrogênio ligado ao C-3, sendo que no espectro de RMN de ${ }^{13} \mathrm{C}$ o sinal correspondente a este carbono mostra sinal entre $\delta 90,0$ e $\delta$ $135,0 .{ }^{42}$ A substância 2 foi identificada como uma C-glicosilflavonaO-glicosídeo, vitexina 2"'-O- $\beta$-D-glucopiranosídeo, ${ }^{43,44}$ e as substâncias 3 e 4 foram identificadas como C-glicosilflavonas, apigenina-8-C- $\beta$ D-glucopiranosídeo e orientina 8-C- $\beta$-D-glucopiranosídeo, respectivamente. $^{45,46}$

Os estudos fitoquímicos de espécies de Piperaceae têm mostrado o acúmulo de uma grande variedade de flavonóides, especialmente com o anel B não substituído. ${ }^{47}$ Entretanto, a presença de flavonóides C-glicosilados é rara. ${ }^{47} \mathrm{~A}$ substância 4, orientina 8-C- $\beta$-D-glucopiranosídeo, já foi previamente isolada de Piper solmsianum e P. lhotzyanum. ${ }^{47,48}$ Derivados C-glicosilados da vitexina, tais como a vitexina-C- $\alpha-\mathrm{L}-$ raminose- $(1 \rightarrow 2)-\beta$-D-glucopiranosídeo, vitexina-C- $\alpha$-L-arabinosídeo e vitexina-C- $\beta$-glucopiranosídeo, foram descritos em Piper methysticum..$^{49}$ Entretanto, a vitexina 2"-O- $\beta$-D-glucopiranosídeo (2) está sendo descrita pela primeira vez neste gênero. Até o momento, derivados da apigenina não foram também descritos em espécies de Piperaceae.

A substância 6 foi identificada como sendo a 5-hidroxi-7,3',4'trimetoxi-flavona, isolada previamente em Piperaceae do extrato de sementes de Piper sylvaticum. ${ }^{42,50}$ A substância 7 foi identificada como sendo a velutina, previamente isolada dos caules e das folhas de Piper clarkii. ${ }^{42,51}$

As substâncias 5 e 8 foram identificadas como sendo as lignanas sesamina e diidrocubebina, as quais já foram isoladas de várias espécies de Piper, incluindo Piper brachystachyum Wall, P. retrofractum, $P$. peepuloides, $P$. guineense e $P$. cubeba. ${ }^{7,52,53}$

\section{MATERIAL SUPLEMENTAR}

Os espectros de $\mathrm{RMN}$ de ${ }^{1} \mathrm{H}$ e RMN de ${ }^{13} \mathrm{C}$ das substâncias isoladas de Piper umbellata encontram-se disponíveis em http://quimicanova.sbq.org.br, na forma de arquivo pdf, com acesso gratuito.

\section{AGRADECIMENTOS}

Ao CNPq, à CAPES e à FAPESP, incluindo o Programa BIOTA (Instituto Virtual da Biodiversidade - www.biota.org.br) pelos auxílios e bolsas concedidas.

\section{REFERÊNCIAS}

1. Taylor, D. W.; Hickey, L. J.; Plant Systematics and Evolution 1992, 180, 137. 
2. Vásquez-Yánes, C.; Segovia, A. O.; Physiological ecology of plants of the wet tropics, Dr. W. Publishers: The Hage, 1984, p. 37.

3. Simpson, B. B.; Ogorzaly, M. O.; Economic Botany: plants in our world, 2nd ed., McGraw-Hill Inc.: New York, 1995.

4. Debonsi, H. M.; Miranda, J. E.; Bortoli, S. A.; Kato, M. J.; Furlan, M.; Pest Manag. Sci.. 2007, 63, 399

5. Orjala, J.; Erdelmeier, C. A. J.; Wright, A. D.; Rali, T.; Sticher, O.; Phytochemistry 1993, 34, 813 .

6. Monache, F. D.; Compagnone, R. S.; Phytochemistry 1996, 43, 1097.

7. Parmar, V. S.; Jain, S. C.; Bisht, K. S.; Taneja, P.; Jha, A.; Tyagi, O. D.; Prasad, A. K.; Wengel, J.; Olsen, C. E.; Boll, P. M.; Phytochemistry 1997, 46, 597.

8. Benevides, P. J. C.; Sartorelli, P.; Kato, M. J.; Phytochemistry 1999, 52, 339.

9. Martins, R. C., Lago, J. H. G.; Kato, M. J.; Phytochemistry 2003, 64, 667.

10. Felippe, L. G.; Baldoqui, D. C.; Bolzani, V. S.; Kato, M. J.; Guimarães, E. F.; Cicarelli, R. M. B.; Furlan, M.; Phytochemistry 2008, 69, 445.

11. Singh, Y. N.; J. Ethnopharmacol. 1992, 37, 13.

12. Alécio, A. C.; Bolzani, V. S.; Young, M. C. M.; Kato, M. J.; Furlan, M.; J. Nat. Prod. 1998, 61, 637.

13. Navickiene, H. M. D.; Alécio, A. C.; Kato, M. J.; Bolzani, V. S.; Young, M. C. M.; Cavalheiro, A. J.; Furlan, M.; Phytochemistry 2000, 55, 621.

14. Silva, R. V.; Navickiene, H. M. D.; Kato, M. J.; Bolzani, V. S.; Meda, C. I.; Young, M. C. M.; Furlan, M.; Phytochemistry 2002, 59, 521.

15. Dodson, C. D.; Dyer, L. A.; Searcy, J.; Wright, Z.; Letourneau, D. K.; Phytochemistry 2000, 53, 51.

16. Cheng, M.; Lee, S.; Chang, Y.; Wu, S.; Tsai, I.; Phytochemistry 2003 63, 603 .

17. Moreira, D. L.; Guimarães, E. F.; Kaplan, M. A. C.; Phytochemistry 1998, 48, 1075.

18. Baldoqui, D. C.; Kato, M. J.; Cavalheiro, A. J.; Bolzani, V. S.; Young, M. C. M.; Furlan, M.; Phytochemistry 1999, 51, 899.

19. Lago, J. G. L.; Ramos, C. S.; Casanova, D. C. C.; Morandim, A. A.; Bergamo, D. C.; Cavalheiro, A. J.; Bolzani, V. S.; Furlan, M.; Guimarães, E. F.; Young, M. M. C.; Kato, M. J.; J. Nat. Prod. 2004, 67, 1783.

20. Morandim, A. A.; Bergamo, D. C. B.; Kato, M. J.; Cavalheiro, A. J.; Bolzani, V. S.; Furlan, M.; Phytochem. Anal. 2005, 16, 282.

21. Salazar, K. J. M.; Paredes, G. E. D.; Lluncor, L. R.; Young, M. C. M.; Kato, M.; Phytochemistry 2005, 66, 573.

22. Lago, J. H. G.; Young, M. C. M.; Reigada, J. B.; Soares, M. G.; Roesler, B. P.; Kato, M. J.; Quim. Nova. 2007, 30, 1222.

23. Danelutte, A. P.; Lago, J. H. G.; Young, M. C. M.; Phytochemistry 2003, $64,555$.

24. Kato, M. J.; Furlan, M.; Pure Appl. Chem. 2007, 79, 529.

25. Jaramillo, M. A.; Manos, P. S.; Zimmer, E. A.; Int. J. Plant Sci. 2004, 165,403

26. Wanke, S.; Samain, M. S.; Vanderschaeve, L.; Mathieu, G.; Goetghebeur, P.; Neinhuis, C.; Plant Biol. 2006, 8, 93.

27. Quijano-Abril, M. A.; Callejas-Posada, R.; Miranda-Esquivel, D. R.; $J$. Biogeografic. 2006, 33, 1266.

28. Wanke, S.; Jaramillo, M. A.; Borsch, T.; Samain, M.; Quandt, D.; Neinhuis, C.; Mol. Phylogenet. Evol. 2007, 42, 477.
29. Kijjoa, A.; Giesbrecht, A. M.; Akiesue, M. K.; Gottlieb, O. R.; Gottlieb, H. E.; Planta Med. 1980, 39, 85.

30. Amorim, C. Z.; Flores, C. A.; Gomes, B. E.; Marques, A. D.; Cordeiro, R. S. B.; J. Ethnopharmacol. 1988, 24, 101.

31. Ferreira-da-cruz, M. F.; Adami, Y. L.; Espínola-mendes, E. C.; Figueiredo, M. R.; Daniel-ribeiro, C. T; Exp. Parasitol. 2000, 94, 243.

32. Desmachelier, C.; Slowing, K.; Ciccia, G.; Fitoterapia 2000, 71, 556.

33. Ropke, C. D.; Kaneko, T. M.; Rodrigues, R. M.; Silva, V. V; Barros, S.; Sawada, T. C. H.; Kato, M. J.; Barros, S. B. M.; Int. J. Pharm. 2002, 249, 109.

34. Felzenszwalb, I.; Valsa, J. O.; Araujo, A. C.; Alcantara, R. G.; Braz. J. Med. Biol. Res. 1987, 20, 403.

35. Desmachelier, C.; Barros, S.; Repetto, M.; Latorre, L. R.; Kato, M. J.; Coussio, J.; Ciccia, G.; Planta Med. 1997, 63, 567.

36. Bergamo, D. C. B.; Kato, M. J.; Bolzani, V. S.; Furlan, M.; J. Braz. Chem. Soc. 2005, 16, 1406.

37. Bergamo, D. C.; Kato, M. J.; Bolzani, V. S.; Furlan, M.; Br PI 070.02386, 2007.

38. Bhakuni, D. S.; Joshi, P. P.; Uprety, H.; Kapil, R. S.; Phytochemistry 1974, 13, 2541.

39. Otsuka, H.; Yao, M.; Kamada, K.; Takeda, Y.; Chem. Pharm. Bull. 1995, $43,754$.

40. Çaliş, I.; Kuruuzum-Uz, A.; Lorenzetto, P. A.; Ruedi, P.; Phytochemistry 2002, 594, 451.

41. Masuoka, C.; Ono, M.; Ito, Y.; Nohara, T.; Food Sci. Technol. Res. 2003, 9, 197.

42. Agrawal, P. K.; Bansal, M. C.; Carbon-13 NMR of flavonoids, Elsevier: New York, 1989.

43. Markham, K. R.; Webby, R. F.; Vilain, C.; Phytochemistry 1984, 23 , 2049.

44. Pauli, G. F.; Junior, P.; Phytochemistry 1995, 38, 1245.

45. Nawwar, M. A. M.; El-mousallamy, A. M. D.; Barakat, H. H.; Buddrus, J.; Linscheid, M.; Phytochemistry 1989, 28, 3201.

46. Hörhammer, L.; Wagner, H.; Gloggengiesser, G.; Arch. Pharm. 1958, 291, 126.

47. Moreira, D. L.; Guimarães, E. F.; Kaplan, M. A. C.; Phytochemistry 2000, 55,783

48. Campos, M. P.; Cechinel Filho, V.; Silva, R. Z.; Yunes, R. A.; Zacchino, S.; Juarez, S.; Bella-Cruz, R. C.; Bella-Cruz, A.; Biol. Pharm. Bull. 2005, 28, 1527 .

49. Jhoo, J. W.; Ang, C. Y. W.; Heinze, T. M.; Deck, J.; Schnackenberg, L. K.; Beger, R. D.; Dragull, K.; Tang, C. S.; Food Chem. Toxicol. 2007, 72,120 .

50. Banerji, A.; Das, R.; Indian J. Chem. Sect. B 1977, 15, 495.

51. Jensen, S.; Olsem, C. E.; Tyagi, O. D.; Boll, P. M.; Hussaini, f. A., Gupta, S.; Bisht, K. S.; Parmar, V. S.; Phytochemistry 1994, 36, 789.

52. Parmar, V. S.; Jain, S. C.; Gupta, S.; Talwar, S.; Raiwanshi, V. K.; Kumar, R.; Azim, A.; Malhortra, S.; Kumar, N.; Jain, R.; Sharma, N. K.; Tyagi, O. D.; Lawrie, S. J.; Erringtpn, W.; Howarth, O. W.; Olsen, G. E.; Singh, S. K.; Wengel, J.; Phytochemistry 1998, 49, 1069.

53. Elfahmi; Ruslan, K.; Batterman, S.; Rein, B.; Kayser, O.; Woerdenbag, H. J.; Quax, W. J.; Biochem. System. Ecol. 2007, 35, 397. 


\section{FLAVONAS, LIGNANAS E TERPENO DE Piper umbellata (PIPERACEAE)}

Debora Cristina Baldoqui, Vanderlan da S. Bolzani e Maysa Furlan*

Instituto de Química, Universidade Estadual Paulista, CP 355, 14800-900 Araraquara - SP, Brasil

Massuo J. Kato

Instituto de Química, Universidade de São Paulo, CP 26077, 05599-970 São Paulo - SP, Brasil

Márcia O. M. Marques

Instituto Agronômico de Campinas, 13075-630 Campinas - SP, Brasil

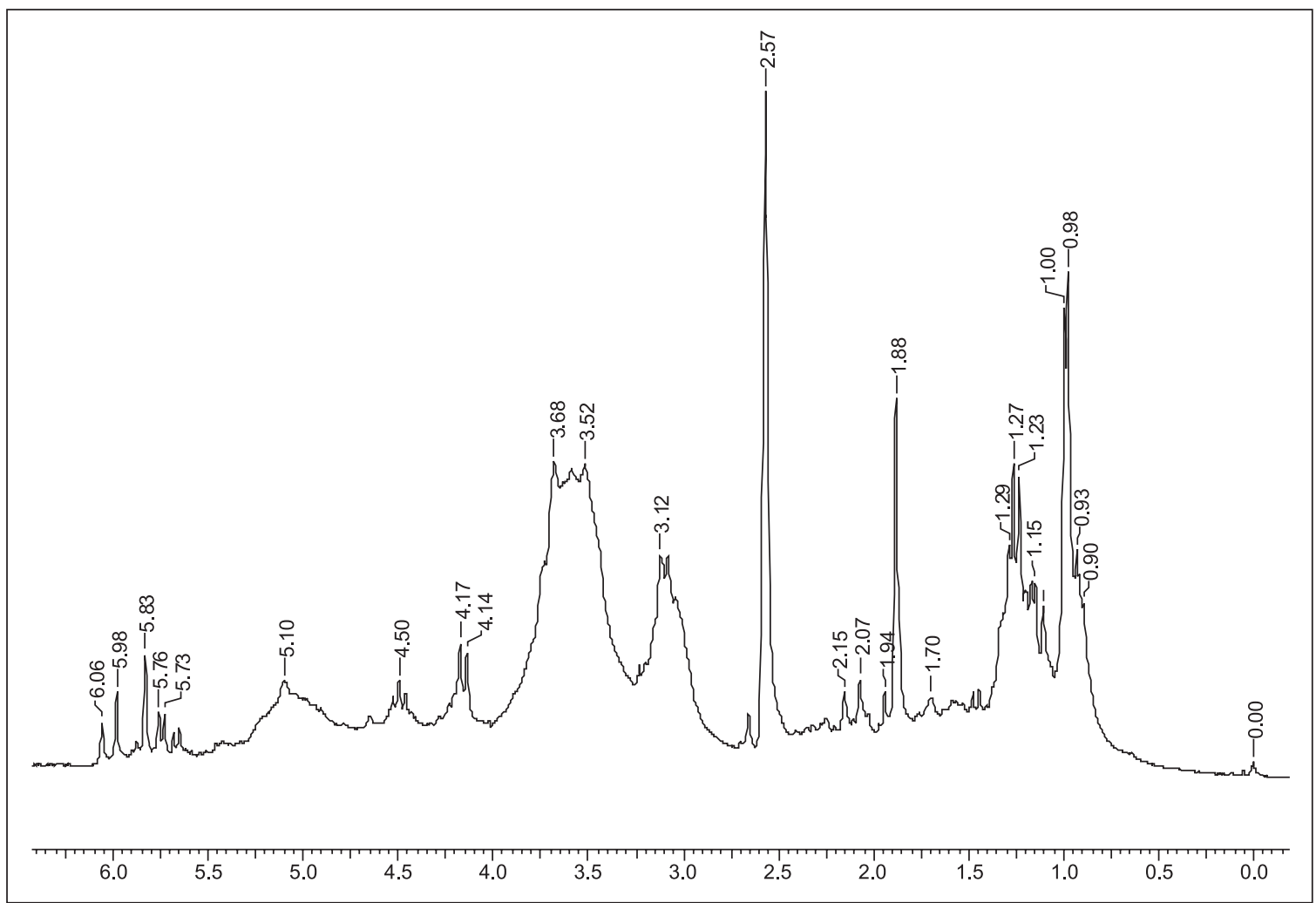

Figura 1S. Espectro de $R M N$ de ${ }^{1} H\left(200 \mathrm{MHz}, \mathrm{DMSO}-d_{6}\right)$ da substância 1

*e-mail: maysaf@iq.unesp.br 


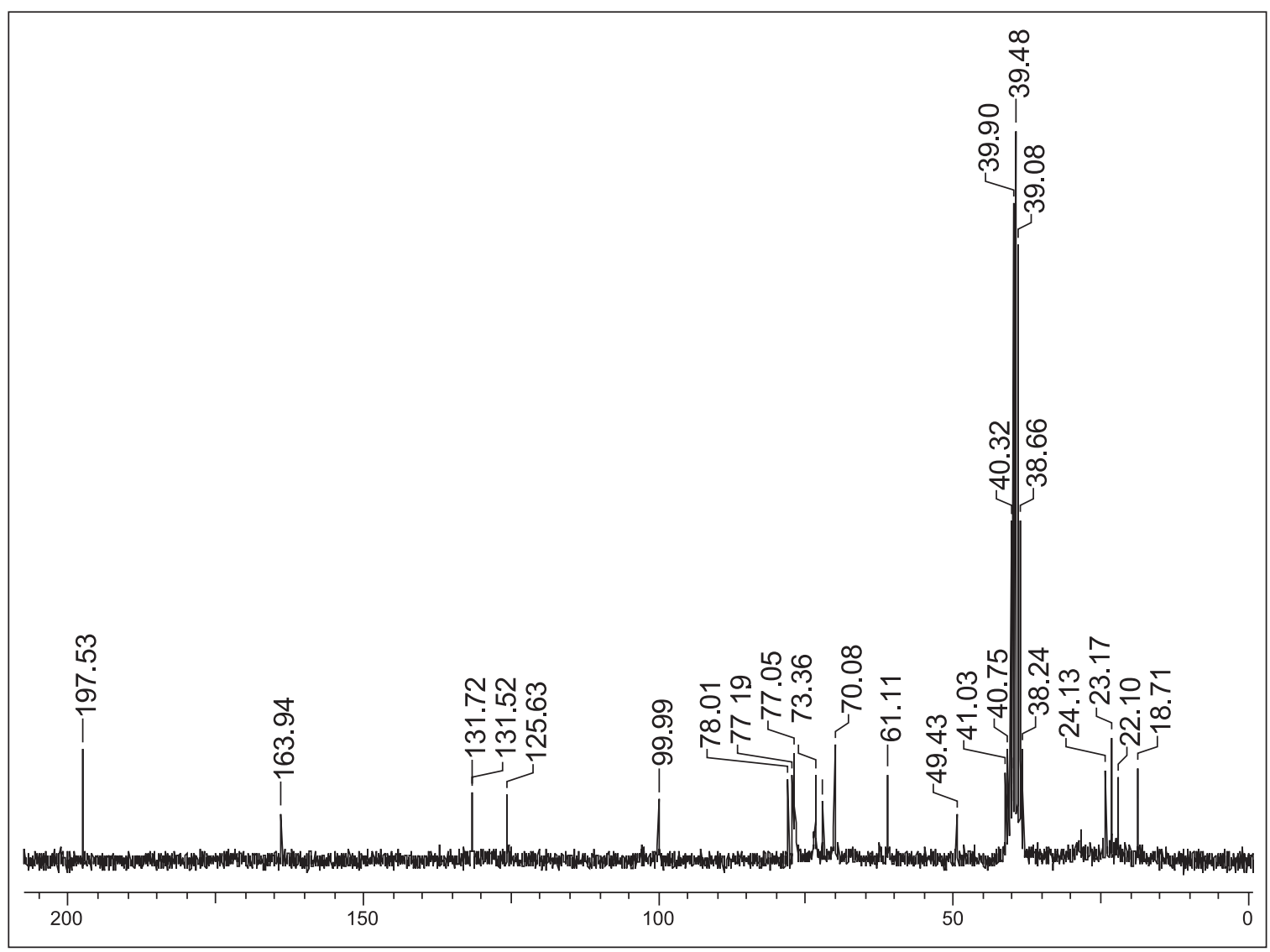

Figura 2S. Espectro de $R M N$ de ${ }^{13} \mathrm{C}\left(50 \mathrm{MHz}, \mathrm{DMSO}-d_{6}\right)$ da substância 1

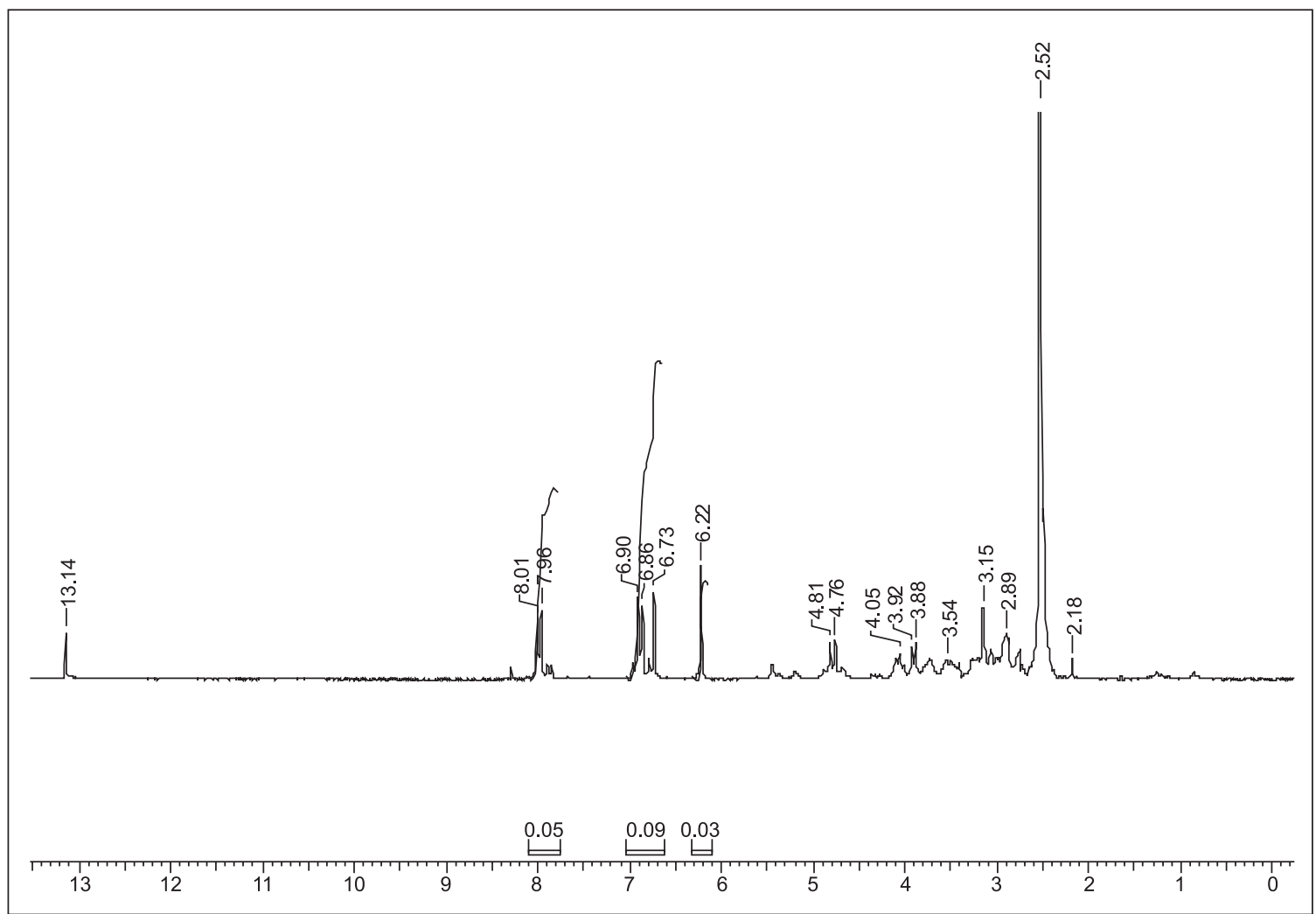

Figura 3S. Espectro de RMN de ${ }^{1} \mathrm{H}(200 \mathrm{MHz}$, DMSO-d $)$ da substância 2 - supressão do pico da água em $\delta 3,3$ 


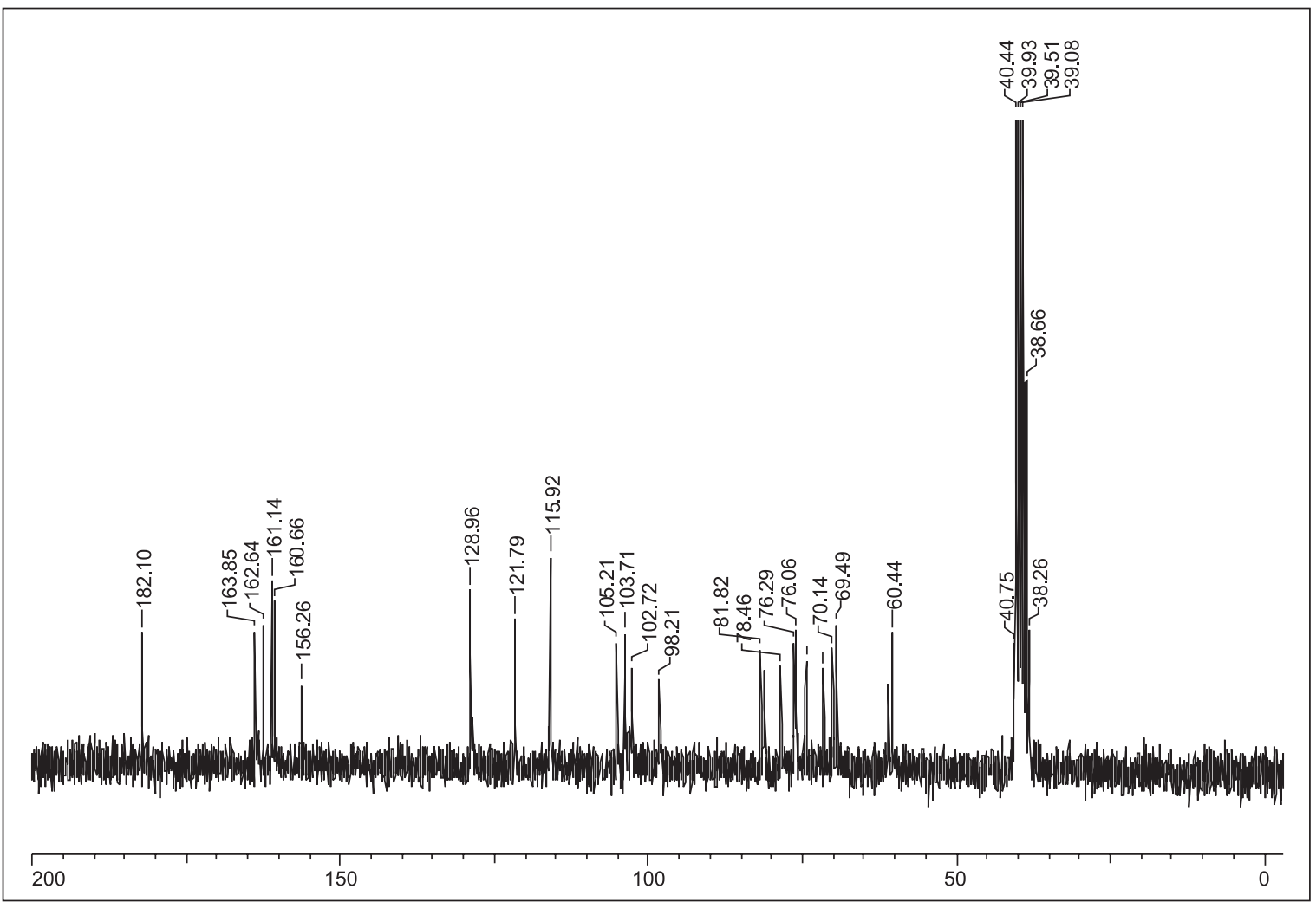

Figura 4S. Espectro de RMN de ${ }^{13} \mathrm{C}\left(50 \mathrm{MHz}, \mathrm{DMSO}-d_{6}\right)$ da substância 2

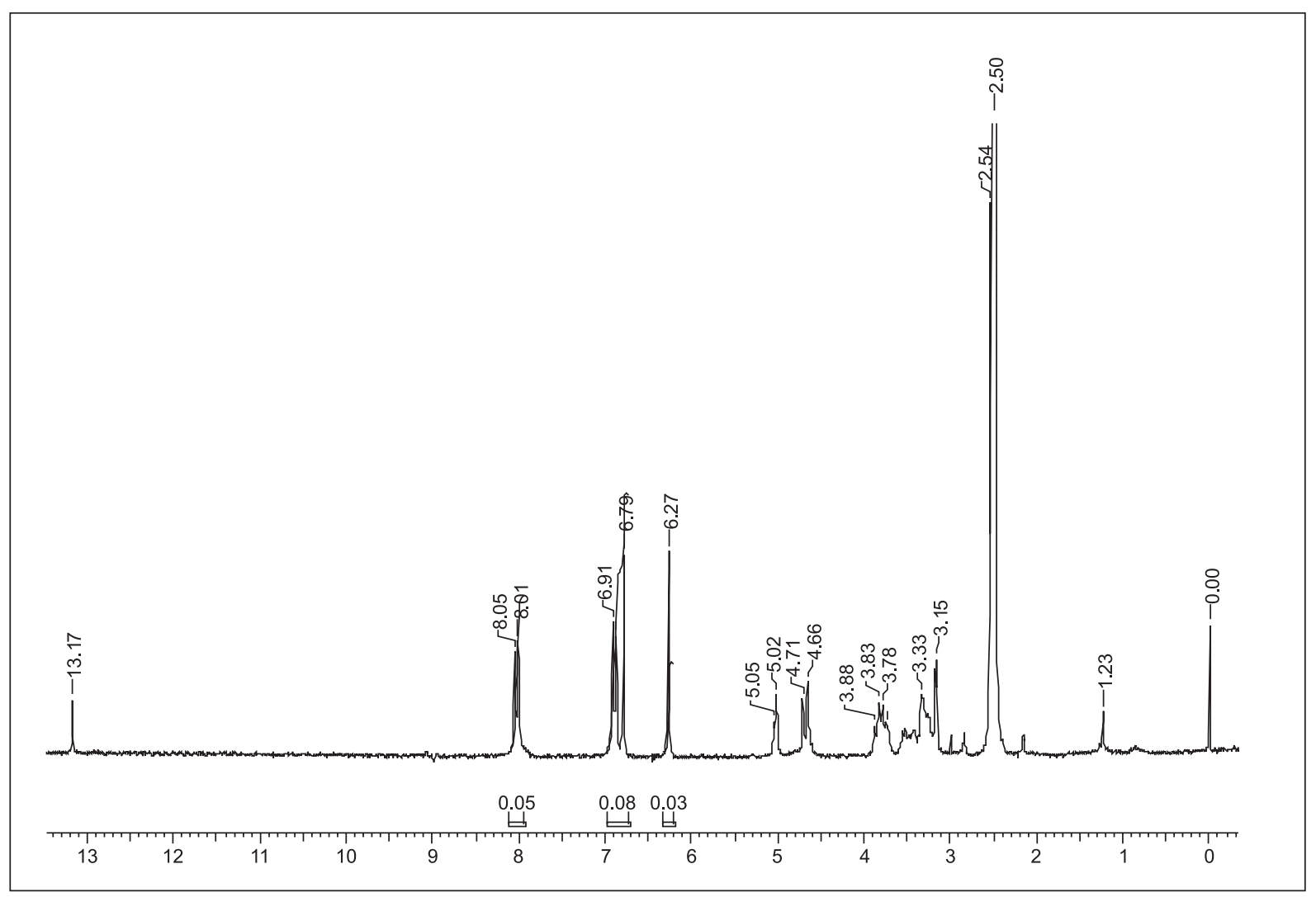

Figura 5S. Espectro de $R M N$ de ${ }^{l} \mathrm{H}\left(200 \mathrm{MHz}, \mathrm{DMSO}-d_{6}\right)$ da substância 3 - supressão do pico da água em 3,3 $\delta$ 


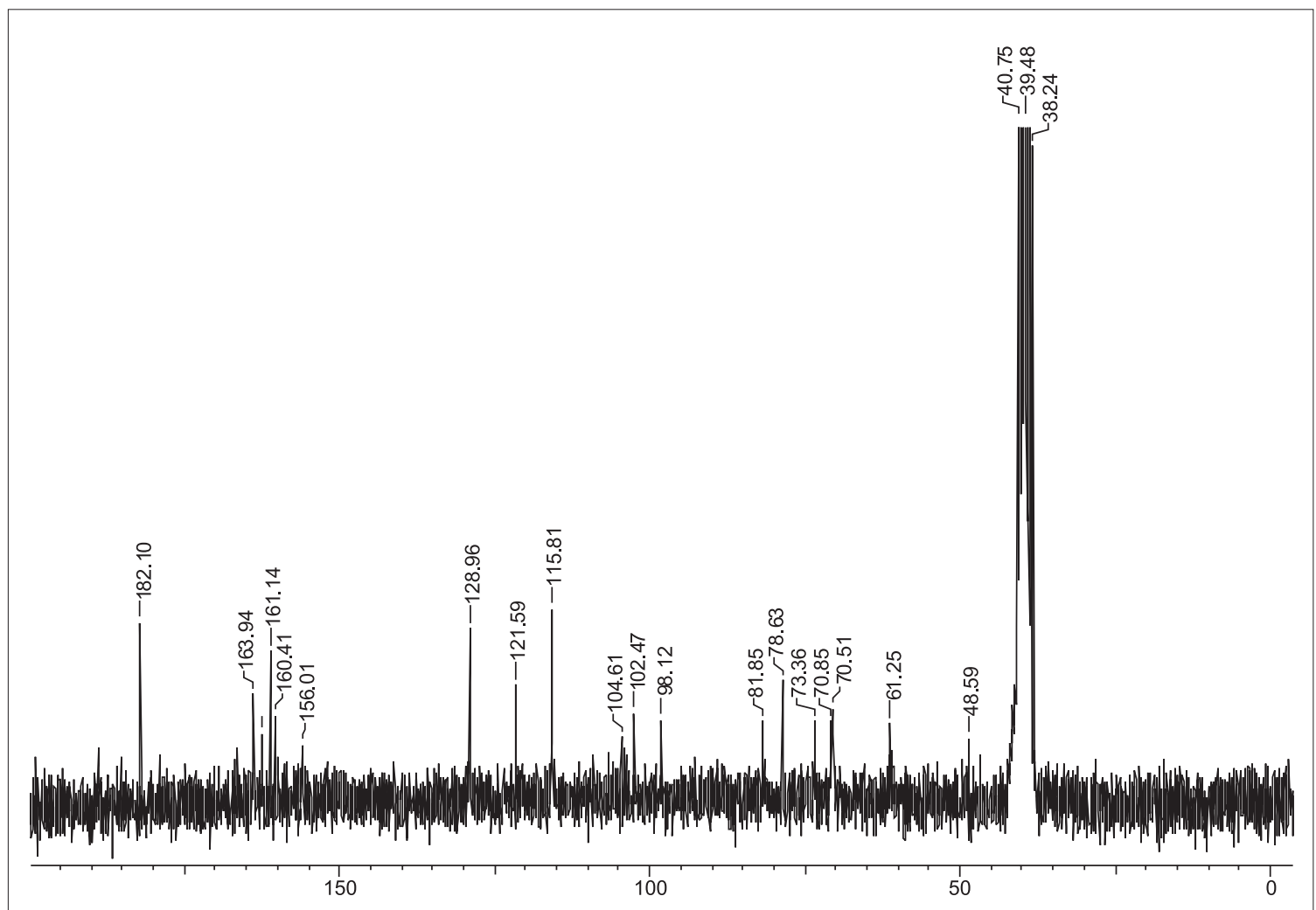

Figura 6S. Espectro de RMN de ${ }^{13} \mathrm{C}\left(50 \mathrm{MHz}, \mathrm{DMSO}-\mathrm{d}_{6}\right)$ de 3

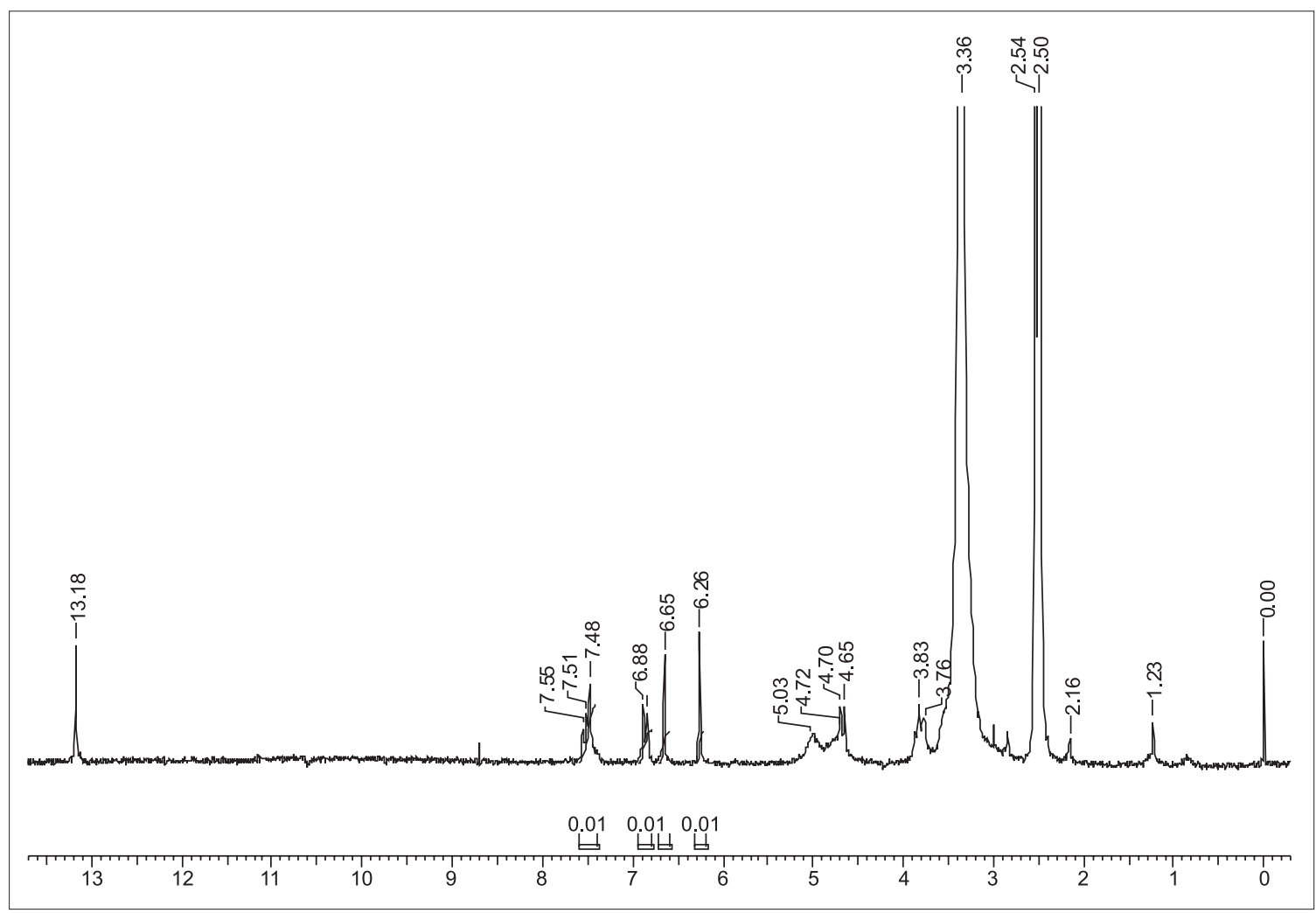

Figura 7S. Espectro de RMN de ${ }^{1} \mathrm{H}\left(200 \mathrm{MHz}\right.$, DMSO-d $\left.{ }_{6}\right)$ da substância 4 


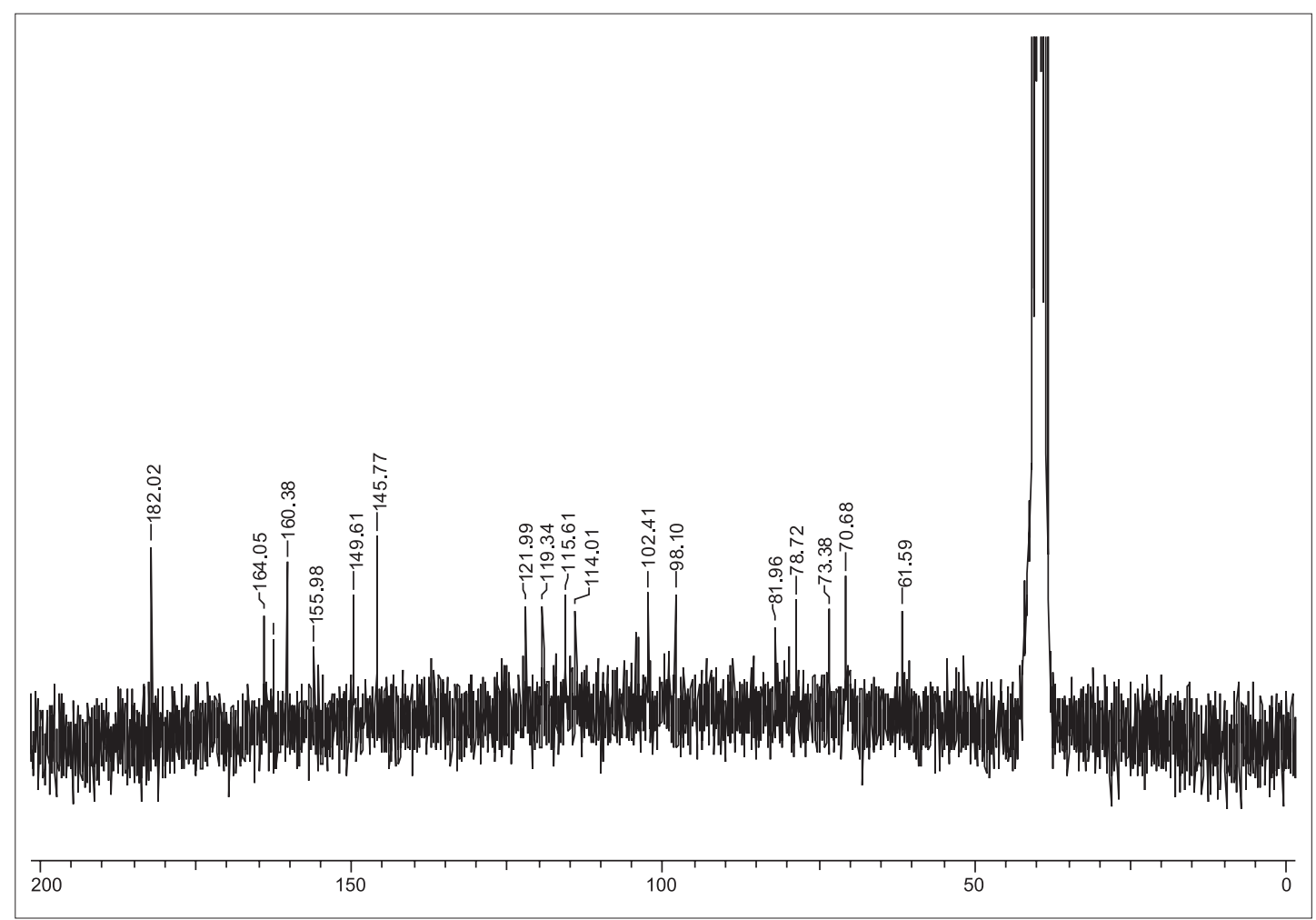

Figura 8S. Espectro de $R M N$ de ${ }^{13} \mathrm{C}\left(50 \mathrm{MHz}, \mathrm{DMSO}-d_{6}\right)$ da substância 4

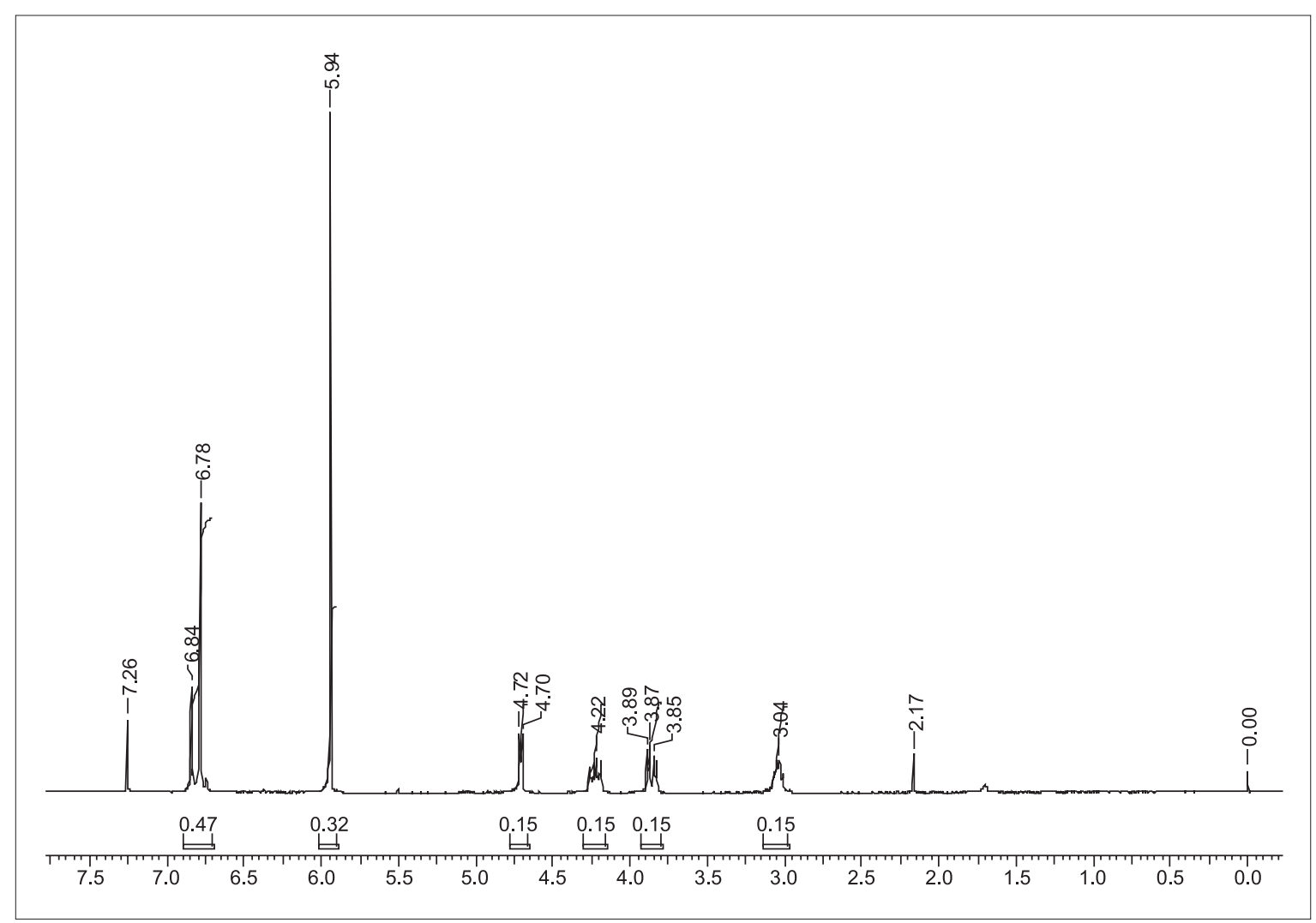

Figura 9S. Espectro de $\mathrm{RMN} \mathrm{de}{ }^{l} \mathrm{H}\left(200 \mathrm{MHz}, \mathrm{CDCl}_{3}\right)$ da substância 5 


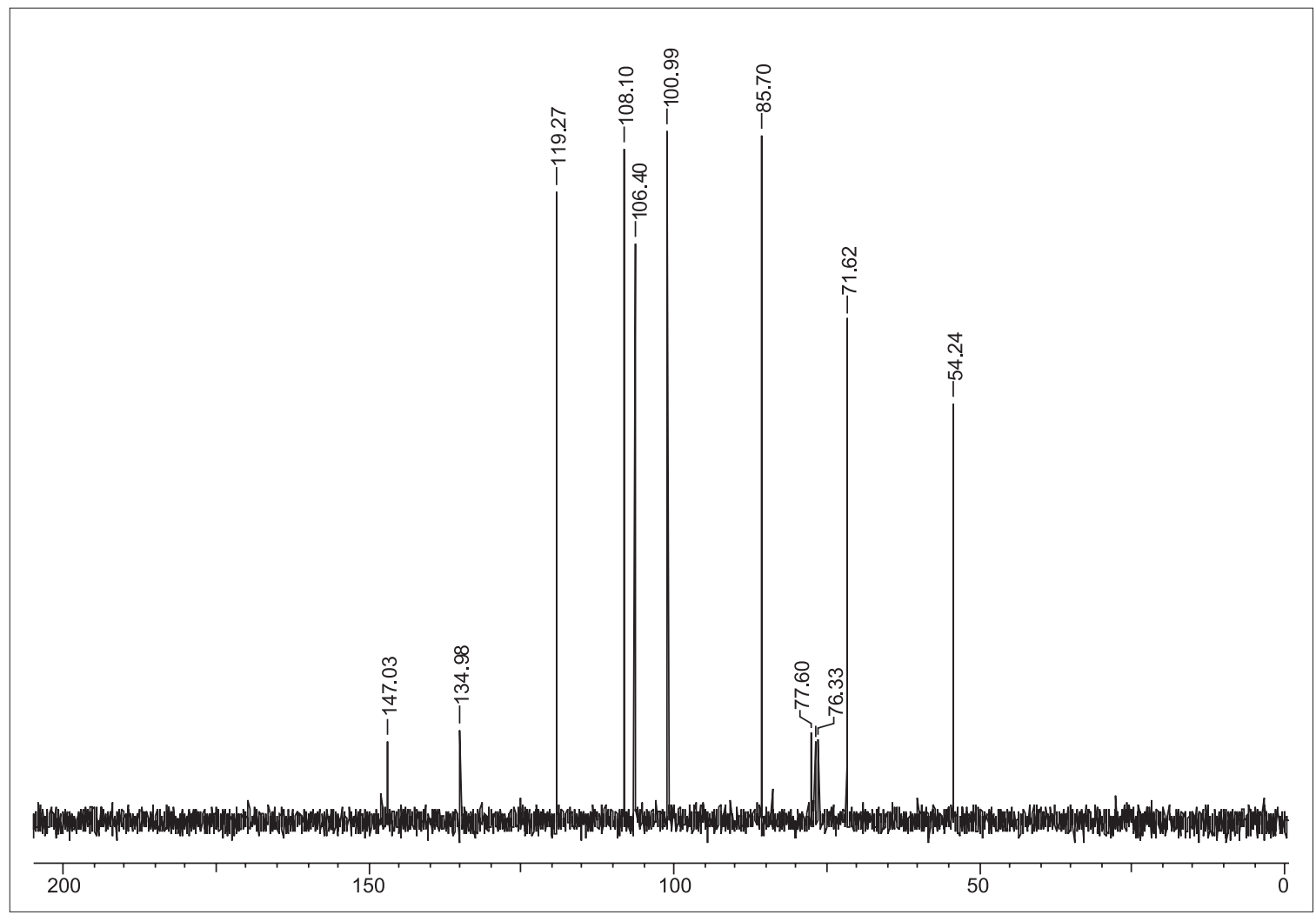

Figura 10S. Espectro de $\mathrm{RMN}$ de ${ }^{13} \mathrm{C}\left(50 \mathrm{MHz}, \mathrm{CDCl}_{3}\right)$ da substância 5

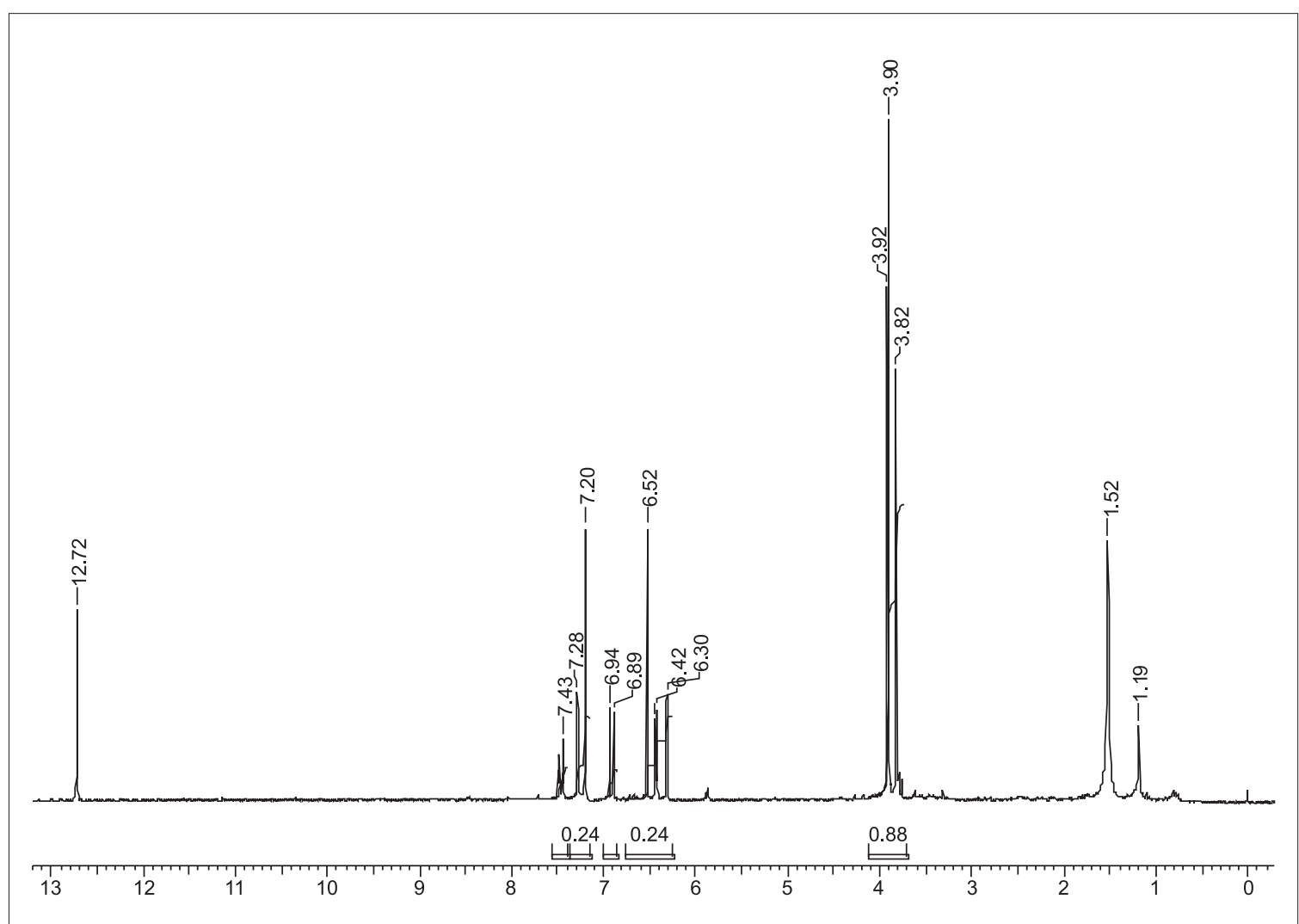

Figura 11S. Espectro de $\mathrm{RMN} \mathrm{de}{ }^{l} \mathrm{H}\left(200 \mathrm{MHz}, \mathrm{CDCl}_{3}\right)$ da substância 6 


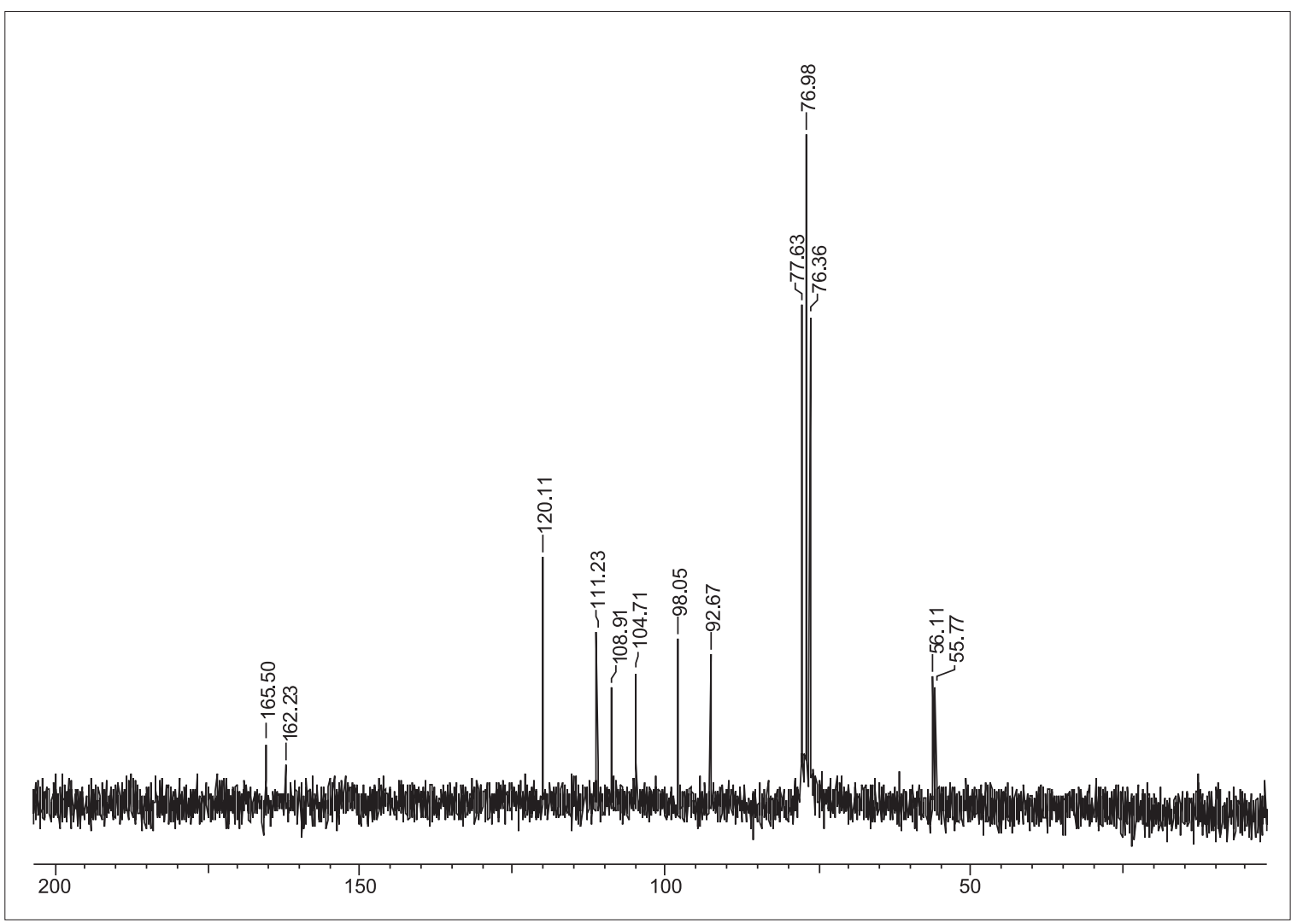

Figura 12S. Espectro de $\mathrm{RMN}$ de ${ }^{13} \mathrm{C}\left(50 \mathrm{MHz}, \mathrm{CDCl}_{3}\right)$ da substância 6

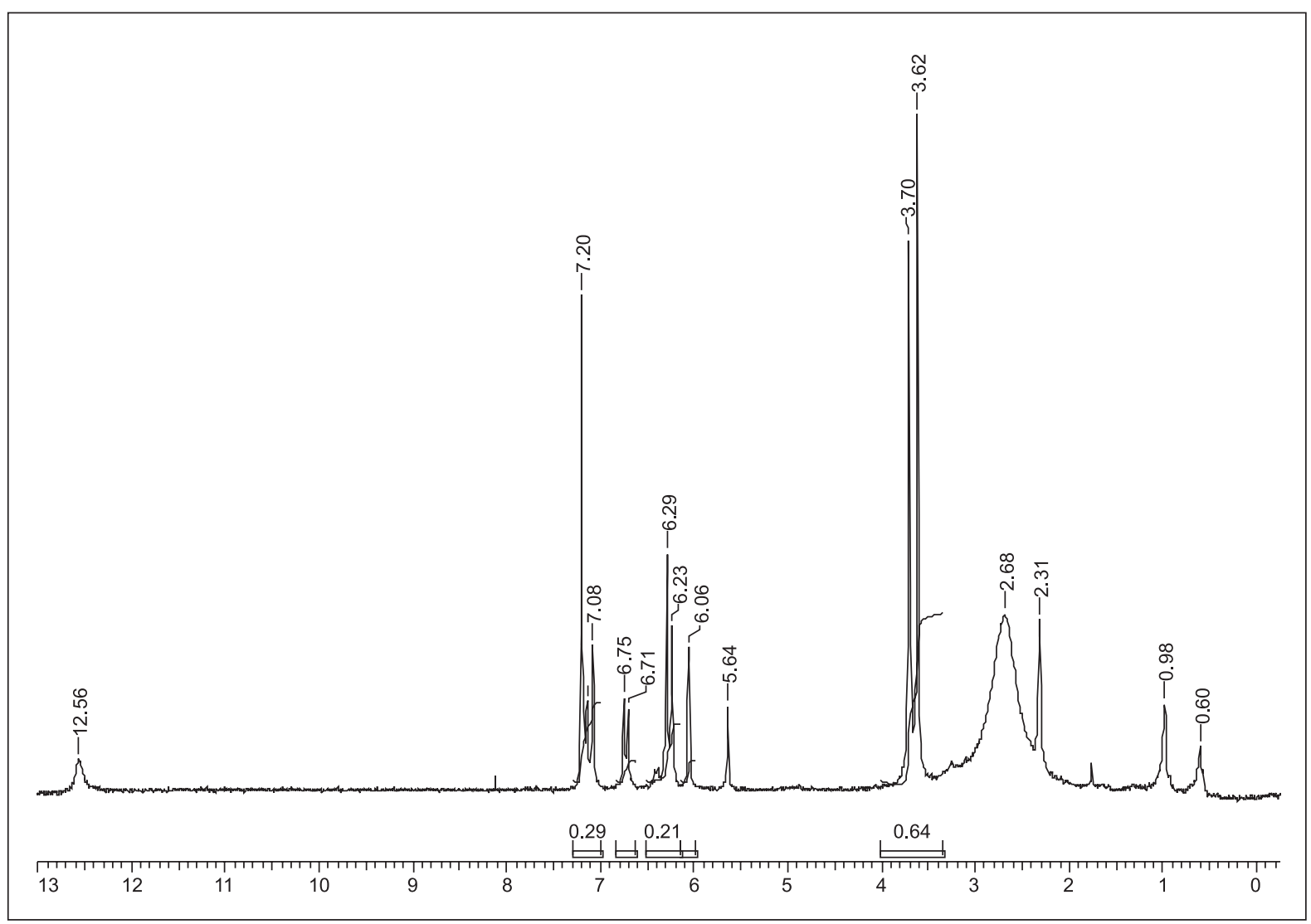

Figura 13S. Espectro de $\mathrm{RMN}$ de ${ }^{l} \mathrm{H}\left(200 \mathrm{MHz}, \mathrm{CDCl}_{3}+\right.$ gotas de DMSO-d $\left.{ }_{6}\right)$ da substância 7 


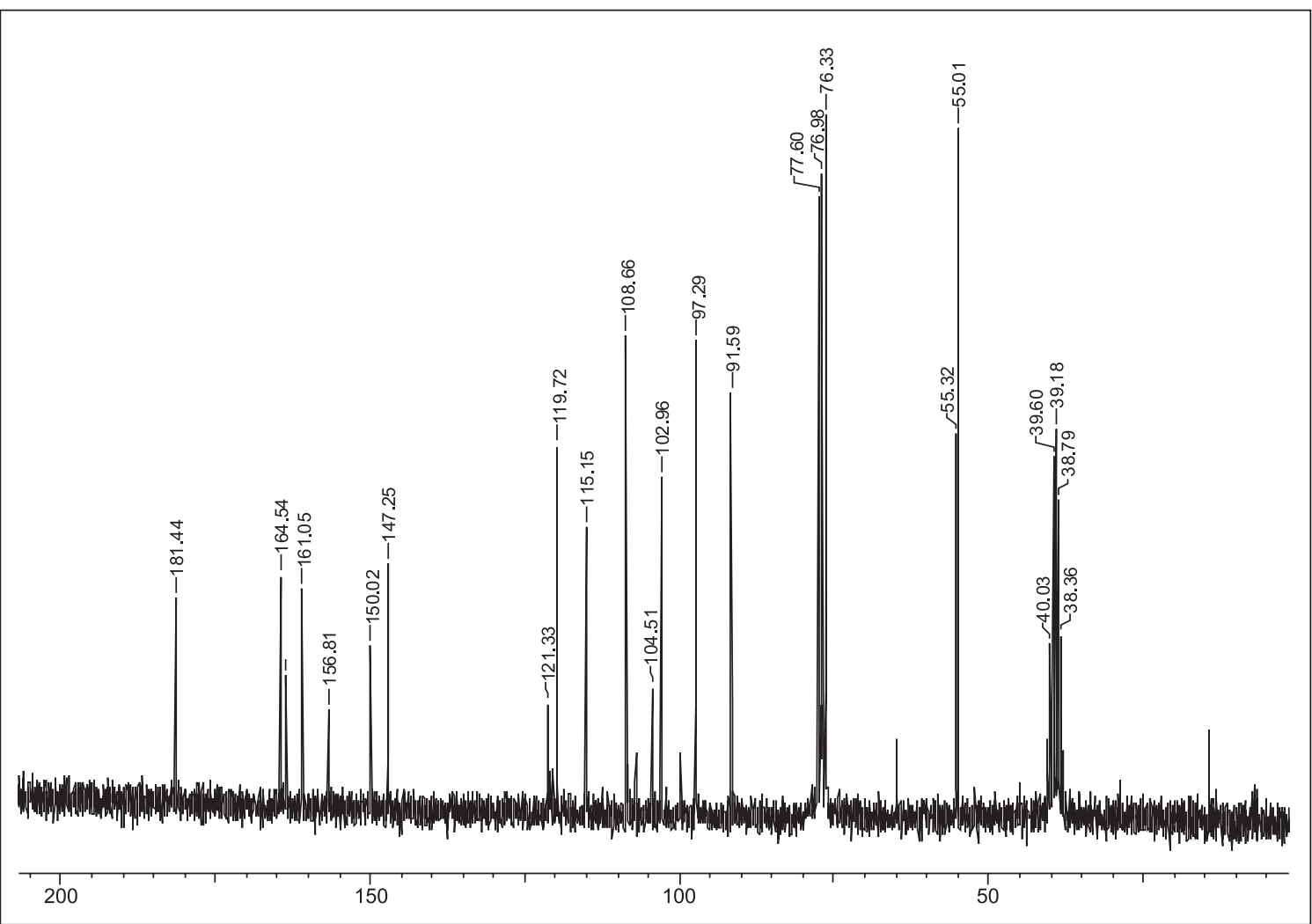

Figura 14S. Espectro de $\mathrm{RMN}$ de ${ }^{13} \mathrm{C}\left(50 \mathrm{MHz}, \mathrm{CDCl}_{3}+\right.$ gotas de DMSO-d $)$ da substância 7

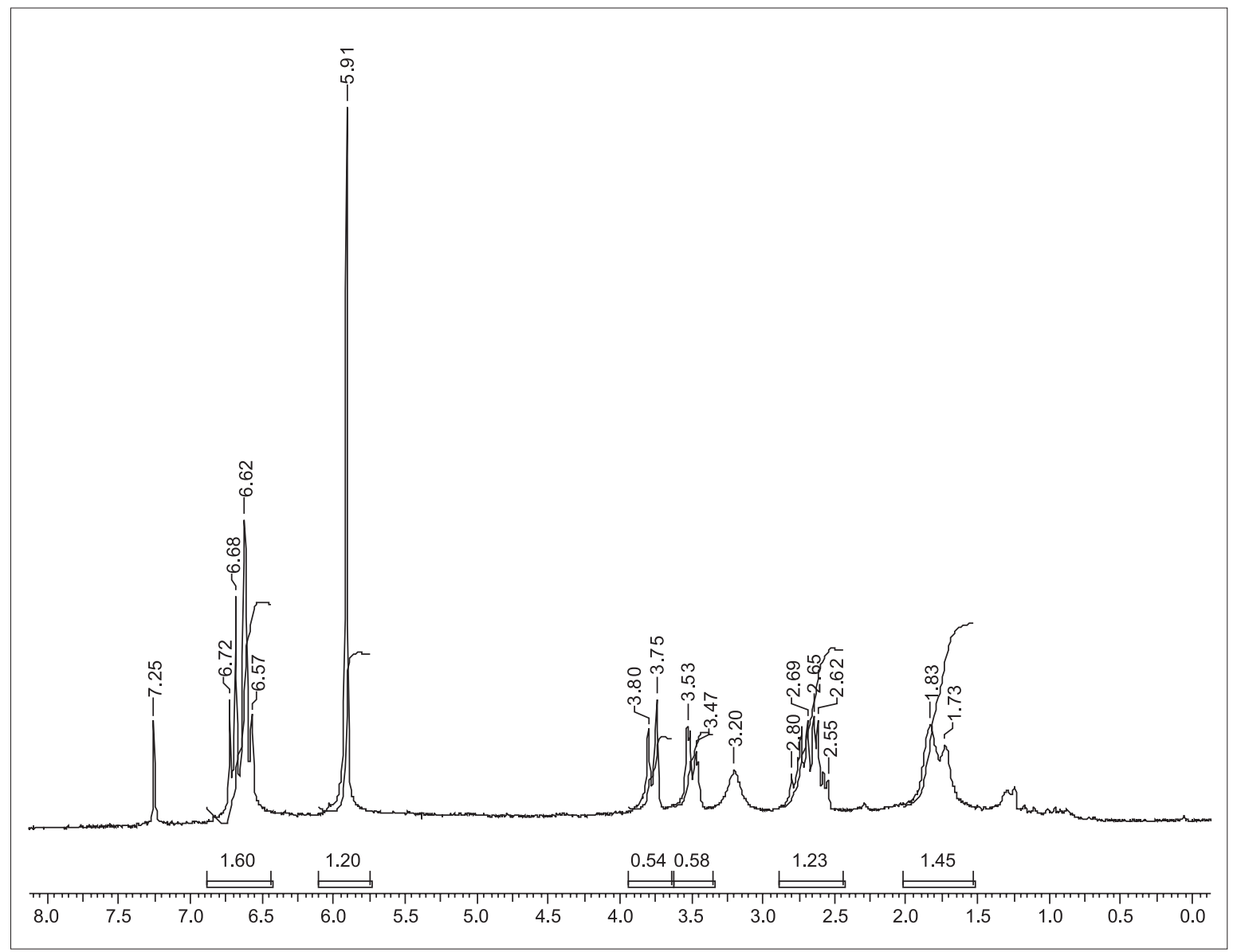

Figura 15S. Espectro de $\mathrm{RMN} \mathrm{de}{ }^{l} \mathrm{H}\left(200 \mathrm{MHz}, \mathrm{CDCl}_{3}\right)$ da substância 8 


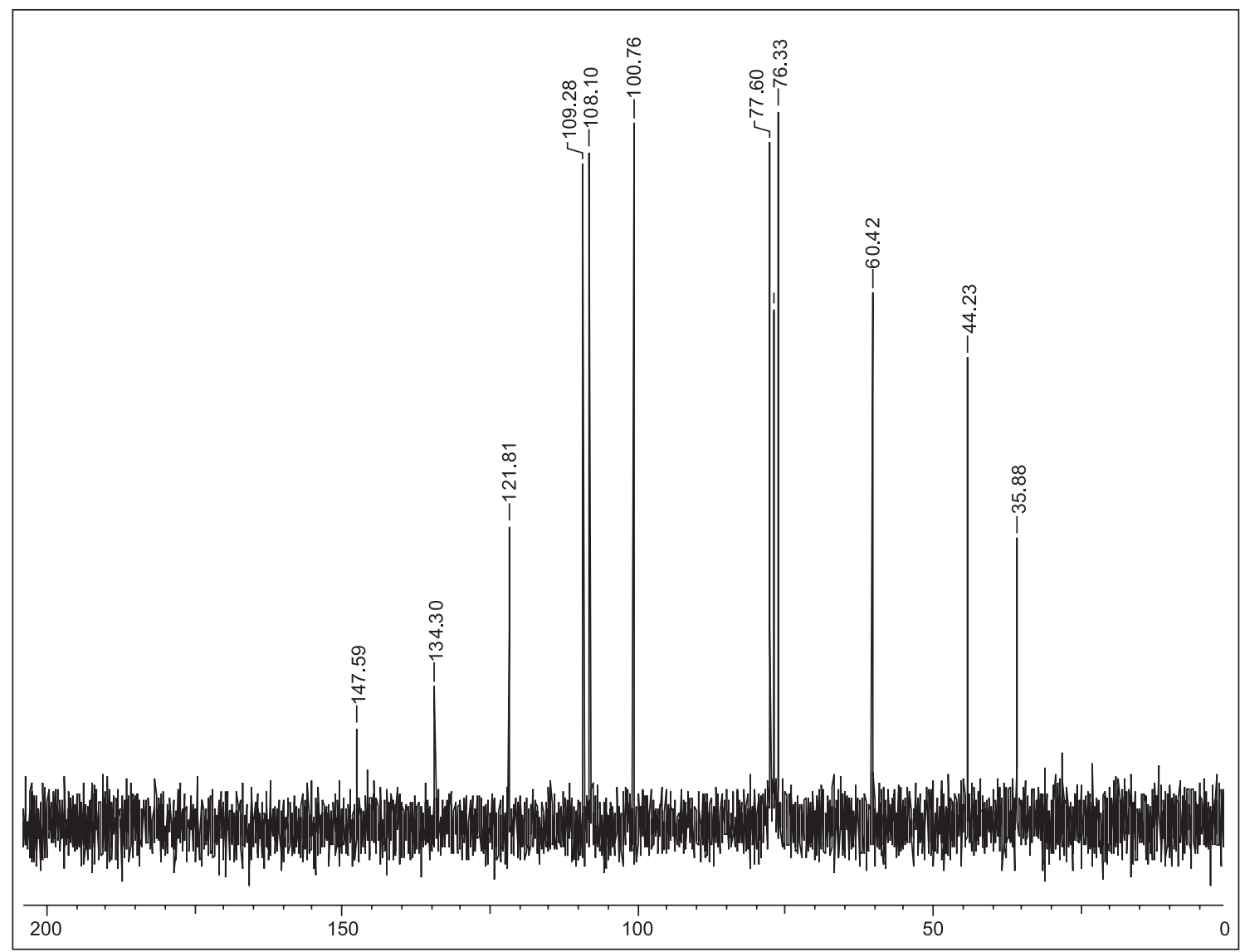

Figura 16S. Espectro de $\mathrm{RMN}$ de ${ }^{13} \mathrm{C}\left(50 \mathrm{MHz}, \mathrm{CDCl}_{3}\right)$ da substância 8

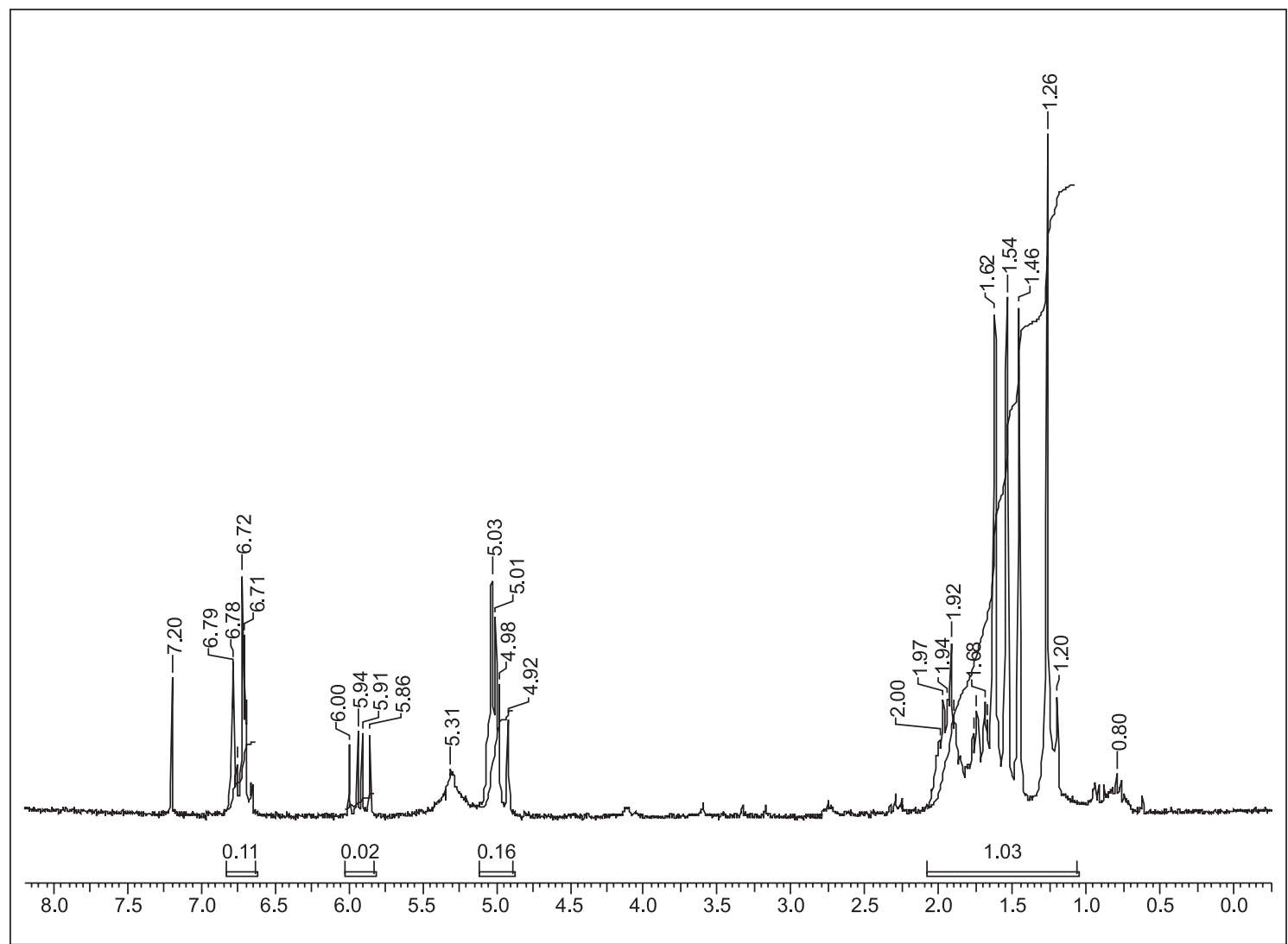

Figura 17S. Espectro de $\mathrm{RMN} \mathrm{de}^{1}\left(200 \mathrm{MHz}, \mathrm{CDCl}_{3}\right)$ da substância 9 


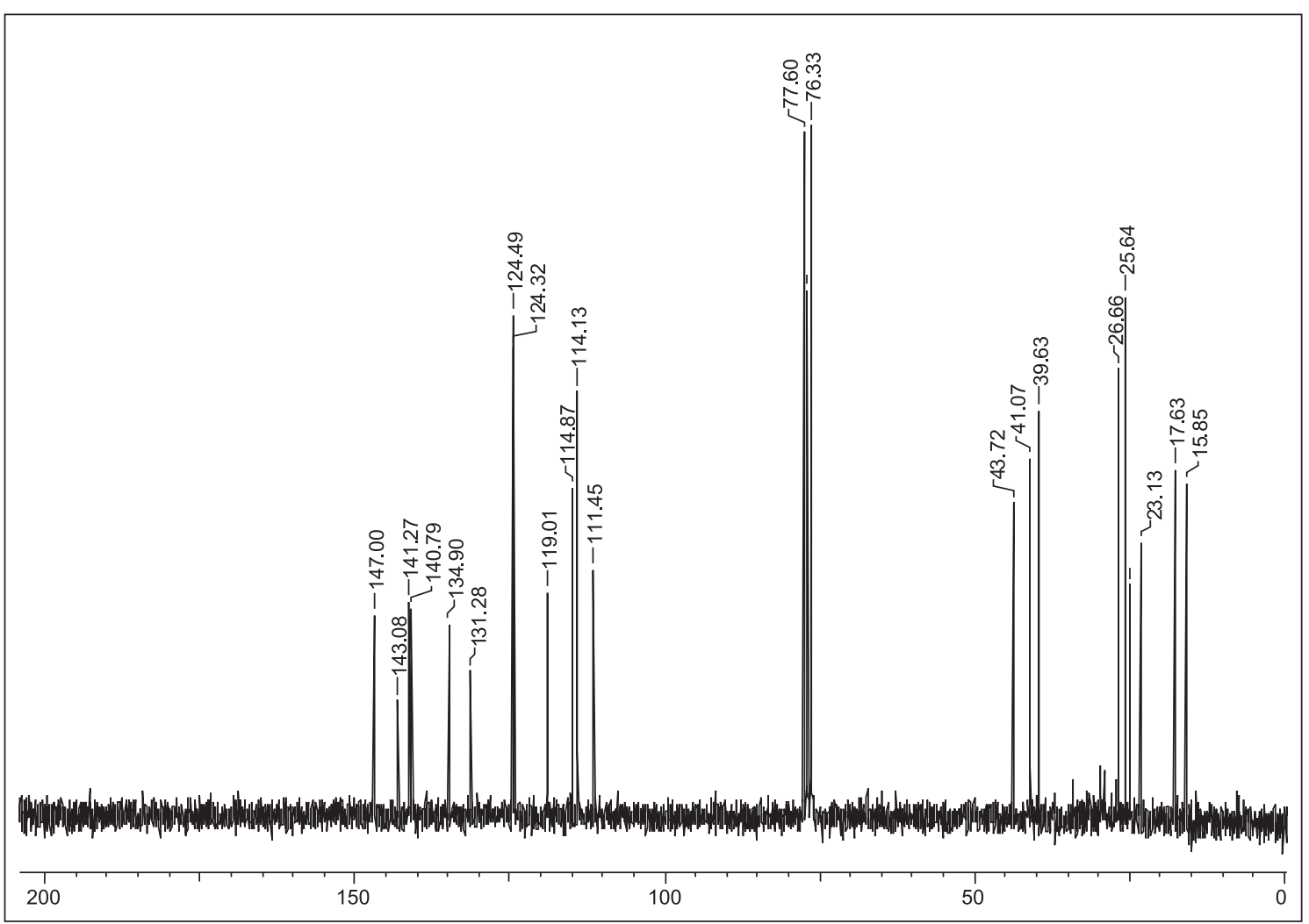

Figura 18S. Espectro de $\mathrm{RMN} \mathrm{de}{ }^{13} \mathrm{C}\left(50 \mathrm{MHz}, \mathrm{CDCl}_{3}\right)$ da substância 9 